\title{
New possibility of the ground state of quarter-filled one-dimensional strongly correlated electronic system interacting with localized spins
}

\author{
Chisa Hotta \\ Aoyama-Gakuin University, 5-10-1, Fuchinobe, Sagamihara, Kanagawa 229-8558 \\ Masao Ogata \\ Department of Physics, University of Tokyo, Hongo, Bunkyo-ku, Tokyo 113-0033
}

Hidetoshi Fukuyama

International Frontier Center for Advanced Materials, IMR, Tohoku University, Miyagi 980-8577

(Dated: September 3, 2018)

\begin{abstract}
We study numerically the ground state properties of the one-dimensional quarter-filled strongly correlated electronic system interacting antiferromagnetically with localized $S=1 / 2$ spins. It is shown that the charge-ordered state is significantly stabilized by the introduction of relatively small coupling with the localized spins. When the coupling becomes large the spin and charge degrees of freedom behave quite independently and the ferromagnetism is realized. Moreover, the coexistence of ferromagnetism with charge order is seen under strong electronic interaction. Our results suggest that such charge order can be easily controlled by the magnetic field, which possibly give rise to the giant negative magnetoresistance, and its relation to phthalocyanine compounds is discussed.
\end{abstract}

PACS numbers: $71.20 . \mathrm{Rv}, 71.30 .+\mathrm{h}, 74.70 . \mathrm{Kn}$

In the quest of conducting states in molecular solids for the past thirty years, charge transfer complexes with the particular 2:1 composition of two different kinds of $\mathcal{H}$ molecules, usually donor and acceptor, have played crucial roles, e.g. $\mathrm{TMTSF}_{2} X$ is the classical example to show the first organic superconductivity(SC) and very rich phases on the plane of pressure and temperature 1 ]. The donor molecules have an average valence of $+1 / 2$ forming a quarter-filled $\pi$ electronic system. There, an interesting competition and coexistence in the types of insulating states are seen under the strong electronic interaction depending on the degree of dimerization; charge ordering $(\mathrm{CO})$ and dimer-Mott expected in the absence of (or under weak) dimerization and at under strong dimerization, respectively [2]. Rich physics in these classes of materials have now been explored. In addition, another family including both quarter-filled $\pi$-electrons and localized spins showed up. For example, in twodimensional $\lambda$-BETS $\mathrm{FeCl}_{4}[\underline{3}]$ and one-dimensional(1D) EDT-TTFVO ${ }_{2} \mathrm{FeBr}_{4}[4$ each acceptor molecule has $S=5 / 2\left(\mathrm{Fe}^{3+}\right)$, the same population number as electrons, whereas in $\mathrm{TPP}\left[\mathrm{Fe}(\mathrm{Pc})(\mathrm{CN})_{2}\right]_{2}$ (phthalocyanine compound) each donor molecule has $S=1 / 2\left(\mathrm{Fe}^{3+}\right)[\underline{5}$, twice the electron number. The combination of localized spins with the strongly interacting quarter-filled electrons offer even more interesting possibilities like field-induced $\mathrm{SC}[\underline{6}]$, ferrimagnetism[4], etc.

In this paper we focus on the 1D TPP $\left[\mathrm{Fe}(\mathrm{Pc})(\mathrm{CN})_{2}\right]_{2}$ and study a simplest model (extended Hubbard-Kondo model) including an antiferromagnetic Kondo coupling, $J$, between the conduction electrons and the localized spins, whose Hamiltonian is given by,

$\mathcal{H}=-\sum_{\langle i j\rangle \sigma} t c_{i \sigma}^{\dagger} c_{j \sigma}+\sum_{\langle i j\rangle} V n_{i} n_{j}+\sum_{j} U n_{j \uparrow} n_{j \downarrow}+\sum_{j} J \overrightarrow{S_{j}} \cdot \overrightarrow{s_{j}} \cdot(1)$ Here, $c_{j \sigma}, n_{j}$, and $\vec{s}_{j}$ are annihilation, number and spin operators at site $j$ for conduction electrons, respectively. $\vec{S}_{j}$ represent spin operators of the localized spins with $S=$ $1 / 2$, and $\langle i j\rangle$ denote the nearest-neighbor(nn) pair sites. We do not take account of the effect of dimerization, which holds in $\mathrm{TPP}\left[\mathrm{Fe}(\mathrm{Pc})(\mathrm{CN})_{2}\right]_{2}$. We focus on the quarter-filling of the conduction electrons where many organic conductors as well as the topic material reside.

For the case with $J=0$, it has been confirmed that the $\mathrm{CO}$ insulating(COI) state is realized at around $U \geq 4 t$ and $V \geq 2 t[7,8]$. On the other hand, usual Kondo lattice model does not include the Coulomb interactions, i.e., $U=V=0$. In this case, when the Kondo coupling is larger than some critical value, $J_{c}(\delta)$, dependent of the electron filling, $\delta$ [9, 10], a ferrimagnetic metallic state is realized, i.e., all the conduction electrons form singlets with the localized spins and the remaining localized spins align in one direction. We call this state as ferromagnetic in the following. This model has already provided some pictures of the interplay of charge and spin degrees of freedom for the heavy fermion systems 11]. Still, the strong electronic interaction adopted in the present model would make the physics richer. In this context, we will study the interplay between the COI and ferromagnetic metal(FM).

First we examine whether the COI state of the extended Hubbard model $(J=0)$ survives in the finite $J$ region. This can be examined by calculating the charge gap, $\Delta_{c}(N)=E_{N}\left(N_{e}+2\right)+E_{N}\left(N_{e}-\right.$ $2)-2 E_{N}\left(N_{e}\right)$, and extrapolating it to infinite system 

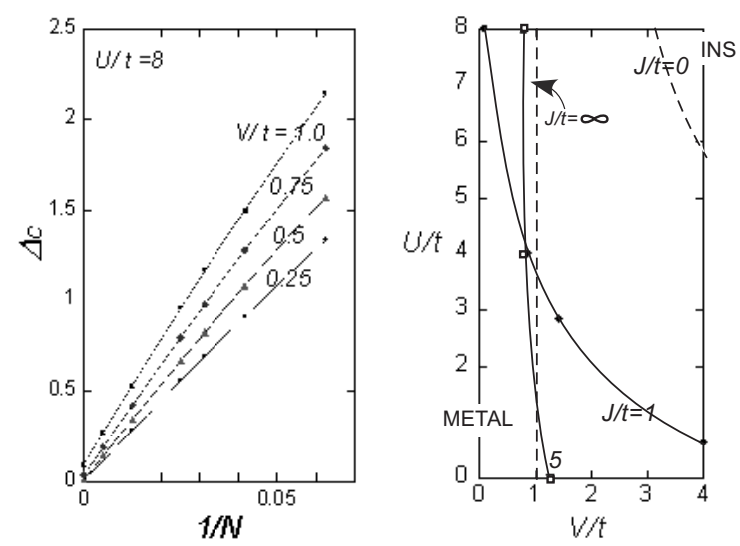

FIG. 1: (a) Charge gap $\Delta_{c}$ as a function of $1 / N$ at fixed value of $J / t=1$ and $U / t=8$ for several choices of $V / t$. Typical truncation error is $10^{-6}-10^{-7}$ with a system size up to $N=$ 200 . Symbols at $1 / N=0$ are the extrapolated values, $\Delta_{c}(\infty)$. (b) Ground state phase diagram on the plane of $U / t$ and $V / t$. The region above the solid (dashed) lines for $J / t=1$ and 5 $(J / t=0, \infty)$ are the COI state where $\Delta_{c}(\infty)$ remains finite.

size. Here, $E_{N}\left(N_{e}\right)$ is the ground state energy of system size $N$ with the electron number $N_{e}$. Figure 1(a) shows some examples of the $N$-dependence of $\Delta_{c}$ obtained by the density matrix renormalization group(DMRG) method 12. We adopt a so-called nonAbelian algorithm [13, 14, 15] with open boundary condition. In this algorithm, we deal with only four states per single site, $\left(s, n_{e}\right)=(1,1),(1 / 2,0),(1 / 2,2),(0,1)$, classified by the total spin, $s$, and the electron number, $n_{e}$, e.g. $(1,1)$ represents a triplet state formed by a localized spin and a conduction electron. The use of this reduced basis provides a dramatic performance of calculation as well as perfect conservation of the $\mathrm{SU}(2)$ symmetry which is quite easily broken in the conventional DMRG.

From Fig凹(a), the charge gap at $N \rightarrow \infty$ is estimated by fitting the data to $\Delta_{c}(N)=\Delta_{c}(\infty)+A / N+B / N^{2}$, where $A$ and $B$ are constants. The metal-insulator (MI) phase diagram at several fixed values of $J / t$ derived from this extrapolation is shown in Fig. 1(b). We use the lowest-energy state irrespective of the total spin. For $J / t=0$, Fig. 1(b) reproduces the previous results by the exact diagonalization in small clusters [7], and the insulating state is the CO state. When $J / t=1$, this COI phase is significantly stabilized. This is rather surprising since the charge degrees of freedom is easily controlled by a small amount of interaction with localized spins. By further increasing $J / t$, the phase boundary asymptotically converges to the $V / t=1$ line in the limit of $J / t=\infty$.

There are several physical reasons of this enhancement of COI state by the Kondo coupling. Basically $J$ introduces a tendency to form a singlet. (I) Firstly, this reduces the effective hopping of the conduction electrons. For example, at $J / t \gg 1$, hopping occurs only when one singlet is broken at a site and then a new singlet is formed on its nn site. In this case, the hopping matrix element is $t_{\text {eff }}=t \times(1 / \sqrt{2})^{2}=t / 2$, and thus $V / t_{\text {eff increases from }}$ the original value of $V / t$, which favors the CO state. (II) Another reason is that the singlet formation disfavors the double occupancy of the conduction electrons. This works cooperatively with $U$. (III) Finally, a singlet state can gain a self-energy by forming a virtual triplet state on the nn sites. This occurs only when the nn site is not occupied by another conduction electron. This plays a similar role as $V$. All these effects extend the $\mathrm{CO}$ region towards the smaller $U / t$ and $V / t$ region.

These phenomena become clearer if we study the strong-coupling region of $J / t \gg 1$ and $U / t \gg 1$. In this region, each single electron forms a singlet with a localized spin on the same site. We can regard this singlet as a vacant site and the remaining unpaired localized spin as "particle" which cannot have double occupancy. Such "particle" is denoted by $d_{j \sigma}$ in the following. The hopping of conduction electrons or singlets is interpreted as that of the "particles" in the inverse direction. For small $t / J$ and $t / U$, we take account of the second order perturbation, and the effective Hamiltonian becomes,

$$
\begin{aligned}
\mathcal{H}_{\mathrm{eff}}= & \sum_{j \sigma}\left(\frac{t}{2} d_{j+1 \sigma}^{\dagger} d_{j \sigma}+\frac{t^{2}}{6 J+4 U} d_{j+2 \sigma}^{\dagger} d_{j \sigma}\left(1-n_{j+1}\right)\right. \\
& \left.+\frac{3 t^{2}}{8 J} d_{j+2 \sigma}^{\dagger} d_{j \sigma} n_{j+1}\right)+ \text { h.c. } \\
- & \sum_{j \tau \tau^{\prime}} \frac{t^{2}}{4 J}\left(d_{j+2 \tau}^{\dagger} d_{j \tau^{\prime}}(\sigma)_{\tau \tau^{\prime}} \cdot S_{j+1}^{d}+\text { h.c. }\right) \\
+ & \sum_{j}\left(V+\frac{3 t^{2}}{2 J}-\frac{2 t^{2}}{3 J+2 U}\right) n_{j}^{d} n_{j+1}^{d}+\text { const., }
\end{aligned}
$$

where $S^{d}$ and $n^{d}$ denote the spin and number operator of the "particles", respectively. The second and the third terms in the first parenthesis give modification of the hopping integral, and the next term with $t^{2} / J$ causes the spin interaction which will be discussed shortly. The last term represents the modification of the $V$ term as mentioned in the reason (III). In the limit of $J=\infty, U=\infty, \mathcal{H}_{\text {eff }}$ is reduced to the $U=\infty$ limit of the extended Hubbard model with $t_{\text {eff }}=t / 2$, because the double occupancy of "particles" is not allowed. Since the nn interaction between "particles" is still equal to $V$, the MI phase boundary is $V / t_{\text {eff }}=2$, i.e., $V / t=1$, consistent with Fig. 1(b).

Let us switch to the magnetic properties. Figure 2(a) shows the ground state phase diagram classified by the total spin, $S$, obtained in our DMRG. The phase transition from a paramagnetic to a ferromagnetic state takes place at around $J / t \sim 1-2$ depending on the values of $U / t$ and $V / t$. At $U=V=0$, a phase transition takes place at $J_{c} / t \sim 1.6$, in concurrence with the results of Ref. 10]. The phase transition is the second order in the sense that the total $S$ changes successively from 0 to $N / 4$. However, the region of intermediate $S$ is small and depends quite much on the numerical condition as in the 


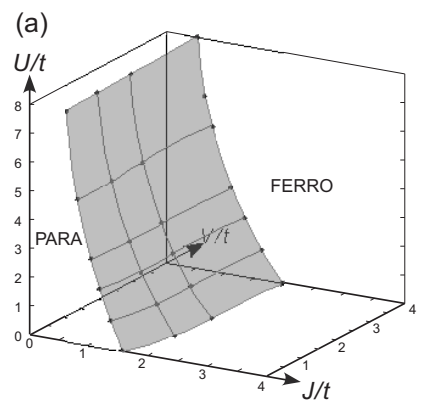

(b)

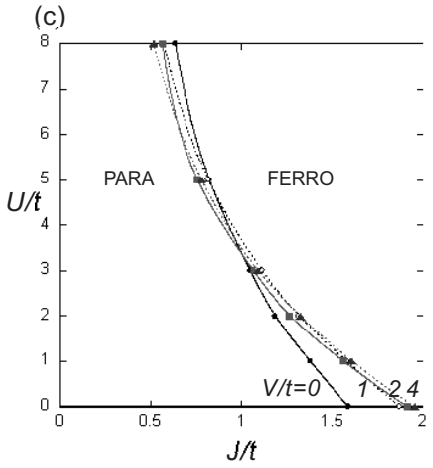

(d)

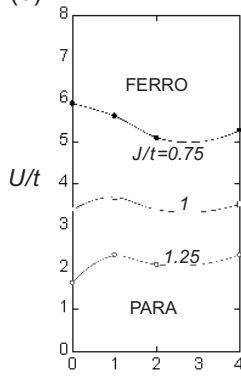

$\mathrm{V} / \mathrm{t}$

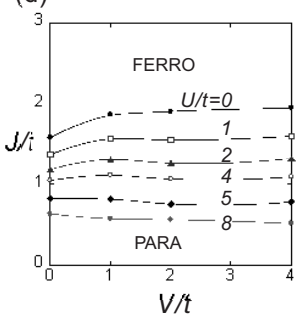

FIG. 2: Ground-state phase diagrams in the space of (a) $U, V$, and $J$, and its cross-section planes (b) $U-V$ for several values of $J / t$, (c) $J-U$ for several values of $V / t$, and (d) $V-J$ for several values of $U / t$. Typical truncation error is $10^{-7}$.

case with $U=V=0[10$, so that we determine the phase boundaries from the energy difference between $S=0$ and $S=N / 4$ states. We also confirm that the spin gap is absent within the numerical error at the representative parameter point of each phases. Figure 2(b) shows the ground state phase diagram on the plane of $U / t$ and $V / t$ which is to be compared with Fig. 囷b). Apparently, the magnetic and MI phase boundaries are not associated with each other and the spin- and charge-degrees of freedom behave independently. Although a charge gap opens above some $V_{c}$, this does not affect the spin degrees of freedom much because the charge gap opens quite slowly.

The detailed cross-section phase diagram on the plane of $U / t$ and $J / t$ is given in Fig. 22(c). The phase boundary moves towards smaller $J / t$ when finite $U / t$ is introduced. This is because the singly occupied states of conduction electrons are stabilized by $U / t$. From Figs. 2(c) and2(d), we can see that the introduction of $V / t$ slightly stabilizes the ferromagnetic phase when $U / t$ is large, whereas the opposite tendency is observed when $U / t$ is small. When $V / t$ is larger than $U / t$, two electrons occupy a single site rather than to stay apart next to each other, and an instability towards superconductivity takes place []. Such double occupancy hinders the ferromagnetism. This is, however, quite an unrealistic situation.

In order to study the ferromagnetism in the large $U / t$ region, it is convenient to use the effective Hamiltonian of eq.(2). As in the large- $U$ Hubbard model, the ground state wavefunction can be expressed as,

$$
\Psi=\psi_{\mathrm{SF}}\left(t_{\mathrm{eff}}, V_{\mathrm{eff}}\right) \Phi_{\mathrm{H}}
$$

where a spinless fermion wavefunction, $\psi_{\mathrm{SF}}\left(t_{\mathrm{eff}}, V_{\mathrm{eff}}\right)$, represents the charge degrees of freedom and $\Phi_{\mathrm{H}}$ the spin degrees of freedom on a squeezed chain [16]. In the limit of $J=\infty, U=\infty$, the spin degrees of freedom are degenerate as in the $U=\infty$ Hubbard model. Then the second-order terms in eq.(2) can be considered as perturbations 17]. The first parenthesis and the last term in eq. (2) modifies $t$ and $V$ of the spinless fermions into $t_{\text {eff }}$ and $V_{\text {eff }}$, respectively. The degeneracy of the spin degrees of freedom is lifted by the remaining terms. These terms give an effective ferromagnetic Heisenberg spin interaction, $J_{\text {eff }} S_{i} \cdot S_{i+1}$, in the squeezed spin chain with

$$
J_{\mathrm{eff}}=\frac{t^{2}}{J}\left\langle d_{j+2}^{\dagger} d_{j} n_{j+1}\right\rangle_{\mathrm{SF}}<0
$$

where $\langle\cdots\rangle_{\mathrm{SF}}$ represents the expectation value in $\psi_{\mathrm{SF}}[16]$. This leads to the ferromagnetic ground state of $\Phi_{\mathrm{H}}$.

Let us discuss the $V$-dependence of $J_{\text {eff }}$. When $V$ or $V_{\text {eff }}$ is introduced it reduces the nn population of "particles", which suppresses $J_{\text {eff }}$. When the $\mathrm{CO}$ becomes dominant, we expect a very small $J_{\text {eff }}$. However, infinitesimal $J_{\text {eff }}$ always stabilizes the ferromagnetic state because of the degenerate perturbation. Actually, we find, $\tilde{J}_{\text {eff }}=-J t^{4} / 2 V^{2}(V+J)^{2}$, in the large $V$-region by considering the fourth order hopping process of Fig. 3. (a); we assume the two localized spins with a singlet in between. The electron hops to its nearest neighbor to form a singlet or a triplet as a virtual state. The triplet formation in the middle of the process (after hopping back to the original site) allows the mixing between left and right neighboring localized spins to give $\tilde{J}_{\text {eff }}$. Among the four different paths in Fig. 3(a), the path including singlts in the first and third virtual state corresponds to the process included in eq. (4). Therefore, the ferromagnetism due to $J_{\text {eff }}$ in eq. (4) naturally continues to that of the large $V$-region. We calculate the $\mathrm{nn}$ and next-nearest neighbor (nnn) correlation functions between charges and localized spins as shown in Fig. 3(b); nn values are suppressed by $V$ in contrast to the nnn ones, which proves the gradual crossover from the ferromagnetism by $J_{\text {eff }}$ to that by $\tilde{J}_{\text {eff }}$.

The ground state phase diagram of eq.(1) is summarized in Fig. 4 at small $J / t$ the phase diagram consists of paramagnetic metal(PM) and paramagnetic $\mathrm{COI}(\mathrm{PCOI})$ at small and large $U / t$ and $V / t$, respectively. With increasing $J / t$, PCOI phase expands. At the same time, the ferromagnetic phases (FM and FCOI) descend significantly from large $U / t$-part at around $J / t \sim 1-2$.

Let us discuss the possible implications of the present theoretical results to experiments in $\operatorname{TPP}\left[M(\mathrm{Pc})(\mathrm{CN})_{2}\right]_{2}$, $M=\mathrm{Fe}$,Co. In the case of Co-salt which does not have localized spins, i.e. $J=0$, a weak sign of $\mathrm{CO}$ is observed in the NQR and the resisitivity shows semiconducting temperature dependence with a small activation energy $\left(\Delta_{a} \sim 10^{-3} \mathrm{eV}\right)$. This indicates that Co-salts are in the $\mathrm{CO}$ region but close to the MI phase boundary as shown in Fig प in view of $U / t \sim 6-8$ and $V / t \sim 3$ with $t \sim 0.1 \mathrm{eV}$ 
(a)

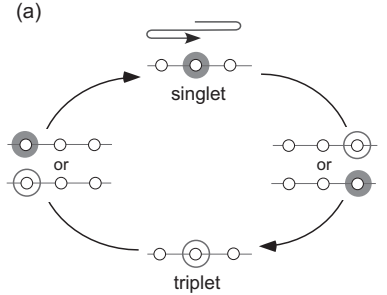

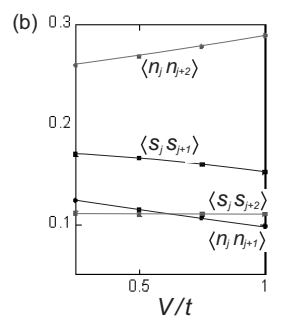

FIG. 3: (a) Fourth order perturbation process at $J, U, V \gg t$ in eq.(1). Filled and open big circles denote the singlet and the triplet, respectively, and the site without them is composed of a free localized spin without electrons. (b) Localized spinspin and charge-charge correlation functions of $\mathrm{nn}$ and $\mathrm{nnn}$ sites averaged over the whole system as a function of $V / t$ at the fixed value of $U / t=8.0$ and $J / t=1.0$.

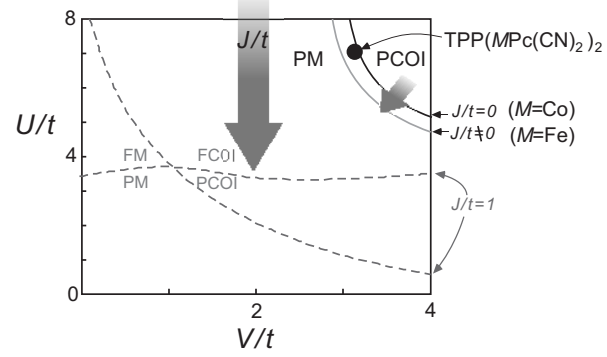

FIG. 4: Summarized phase diagram on the plane of $U / t$ and $V / t$. Black circle represents the possible location of $\mathrm{TPP}\left[M(\mathrm{Pc})(\mathrm{CN})_{2}\right]_{2}(M=\mathrm{Co}, \mathrm{Fe})$.

given by the extended Hückel calculation. The magnetic susceptibility behaves as that of the 1D Heisenberg $S=1 / 2$ spin system. Here, the antiferromagnetic coupling is expected to be several factors larger than the one between spins of completely localized charges $\left(J_{\mathrm{AF}}=t^{4} / U V^{2} \sim 10 \mathrm{~K}\right)$, because of a certain degree of delocalization in the proximity of the MI boundary.

As for the Fe-salt with localized $S=1 / 2$ spins, the resistivity is semiconducting with larger $\Delta_{a} \sim 10^{-2} \mathrm{eV}$, which indicates the stronger $\mathrm{CO}$ in Fe salt than in $\mathrm{Co}$ salt. This is consistent with the present results that the coupling to the localized spins generally enhances the charge gap. The magnetic susceptibility in Fe-salt shows large anisotropy between those $\operatorname{parallel}\left(\chi_{\|}\right)$and perpendicular $\left(\chi_{\perp}\right)$ to the $1 \mathrm{D}$ chain; $\chi_{\|}$behaves similar to the Co-salt case, while $\chi_{\perp}$ obeys the Curie-law at $T>T_{N} \sim 25 \mathrm{~K}$ and suddenly saturates to become weakly $T$-dependent at $T \sim 5 \mathrm{~K}-25 \mathrm{~K}$, followed by a weak ferromagnetism at $T<5 \mathrm{~K}$. Noting that magnetic easy axis of Fe ions is esentilally perpendicular to the $1 \mathrm{D}$ chain, we may assume that $\chi_{\|}$and $\chi_{\perp}$ are originated mainly from the electrons and the Fe spins, respectively. Hence, the similarity of $\chi_{\|}$with that of the Co salt is understood, while the behavior of $\chi_{\perp}$ will be interpreted as follows; the saturation below $T_{N}$ suggests that the antiferromagnetic correlation developes below $T_{N}$, which is due to the onset of $\pi$-d interaction roughly estimated as $J \sim T_{N}$. In our model, this $J / t \sim 0.025$ actually pushes enough the MI phase boundary to the region of smaller $U / t$ and $V / t$ to make Fe-salt a PCOI. Such small $J / t$ does not lead to ferromagnetism which also agrees well with the experiment. We speculate that the weak ferromagnetism at $T \sim 5 \mathrm{~K}$ is due to the canted magnetic moments induced by impurities in the antiferromagnetic background.

Interestingly, in the Fe-salt, a giant negative magnetoresistance (GNMR) is reported below $T \sim 50 \mathrm{~K}[18]$. This will simply be understood in the present framework(eq.(10) as due to the suppression of CO phase. When the magnetic field is applied, the total $S_{z}$ increases and each site will have the larger population of localized $S_{z}=1 / 2$ (without electrons) as well as of $S_{z}=1$ triplets composed of one eletron and a localized spin, whereas the number of singlets with $S_{z}=0$ decreases. Since the electron hopping from $S_{z}=1$-site to $S_{z}=1 / 2$-site gives $t_{\mathrm{eff}}=1$, which is larger than those assosiated with $S_{z}=0$-site $\left(t_{\text {eff }}=t / \sqrt{2}\right.$ or $t / 2), V / t_{\text {eff }}$ is reduced and the charge gap will be suppressed. This process of GNMR is quite different from the conventional double-exchange mechanism 19].

In conclusion, the $\mathrm{CO}$ in the quarter-filled strongly correlated electronic system becomes stable when combined with the localized spins, and this CO could be easily controled by the magnetic field.

[1] D. Jérome, Science 252, 1509, (2004).

[2] H. Seo, C. Hotta, H. Fukuyama, Chem. Rev. 104, 5005, (2004), and the references therein.

[3] H. Kobayashi, A. Kobayashi, T. Cassoux, Chem. Soc. Rev., 29, 325, (2000).

[4] S. Noguchi, A. Matsumoto, T. Matsumoto, T. Sugimoto, T. Ishida, Physica B 346-347 397 (2004).

[5] T. Inabe, H. Tajima, Chem. Rev. 104, 5503 (2004).

[6] S. Uji, H. Shinagawa, C. Terakura, T. Terashima, T. Yakabe, Y. Terai, M. Tokumoto, A. Kobayashi, H. Tanaka, and H. Kobayashi, Nature 410, 908 (2001).

[7] F. Mila and X. Zotos, Europhys. Lett. 24, 133 (1993).

[8] H. Yoshioka, M. Tsuchiizu and Y. Suzumura, J. Phys. Soc. Jpn. 69, 651 (2000).

[9] H. Tsunetsugu, M. Sigrist, and K. Ueda, Phys. Rev. B 47, 8345 (1993).

[10] I. P. McCulloch, A. Juozapavicius, A. Rosengren, M. Gulacsi, Phys. Rev. B 65, 052410 (2002).

[11] G. Aeppli, Z. Fisk, Comments Cond. Mat. Phys. 16, 155 (1992).

[12] S.R. White, Phys. Rev. Lett. 69, 2863 (1992).

[13] I. McCulloch, M. Gulacsi, Europhys. Lett. 57, 852 (2002).

[14] In this algorithm at $S \neq 0$, the newly obtained basis after the truncation is a linear combination of the old basis with several total spins, i.e. the new density matrix has off-block-diagonal elements between different total spins. However, neglecting the off-block-diagonal elements does not alter the results within the numerical error [13].

[15] The number of states at each block, $m$, are kept up to 200 which is enough to suppress the maximum truncation error to less than the order of $10^{-5}-10^{-6}$ in the worst case.

[16] M. Ogata, H. Shiba, Phys. Rev. B41, 2326 (1990). 
[17] H. Shiba, M. Ogata, Int. J. Mod. Phys. B5, 31 (1991).

[18] N. Hanasaki, H. Tajima, M. Matsuda, T. Naito, T. Inabe, Phys. Rev. B 62, 5839 (2000).

[19] A. Moreo, S. Yunoki, E. Dagotto, Science 2832034
(1999); K. Kubo, N. Ohata, J. Phys. Soc. Jpn. 3321 , (1972). 